\title{
Influence of humidity on local polarization reversal in Rb:KTP single crystal
}

Ekaterina V. Shishkina1, Elena V. Pelegova', Mikhail S. Kosobokov', Andrey R. Akhmatkhanov' ${ }^{1}$ Petr V. Yudin ${ }^{2,3}$, Alexandr Dejneka ${ }^{2}$, Vladimir Ya. Shur ${ }^{1 *}$

${ }^{1}$ School of Natural Sciences and Mathematics, Ural Federal University, 620000

Ekaterinburg, Russia

${ }^{2}$ Institute of Physics, Academy of Sciences of the Czech Republic, 18221 Praha 8,

Czech Republic

${ }^{3}$ Kutateladze Institute of Thermophysics, Siberian Branch of Russian Academy of

Science, 630090 Novosibirsk, Russia

Electronic mail: vladimir.shur@urfu.ru 
S1. Simulation of the spatial field distribution produced by a biased tip with a water meniscus

The spatial distribution of the polar component of the electric field $\left(E_{z}\right)$ produced by a biased conductive tip of a scanning probe microscope (SPM) with a water meniscus

(Fig.1a) was simulated by the finite element method using the commercial software COMSOL Multiphysics with AC/DC Electrostatics interface.

The considered SPM tip had a cone-shaped geometry with a semispherical end and a circular disk-type contact area. The height of the tip conical part was $3 \mu \mathrm{m}$, the cone angle was 40 degrees, the sphere radius was $30 \mathrm{~nm}$, and the radius of the contact area was $5 \mathrm{~nm}$. The tip bias voltage was $+150 \mathrm{~V}$. The solid bottom electrode was grounded. The sample thickness was $10 \mu \mathrm{m}$. The radius of the circular shaped water meniscus $\left(R_{w}\right)$ was ranged from 100 to $900 \mathrm{~nm}$. The dielectric constants of the sample and water were 30 and 80 , respectively. The typical spatial distributions of $E_{z}$ at $Z Y$ cross-section and at $10 \mathrm{~nm}$ below the polar surface are presented in Figure $\mathrm{S} 1$. 

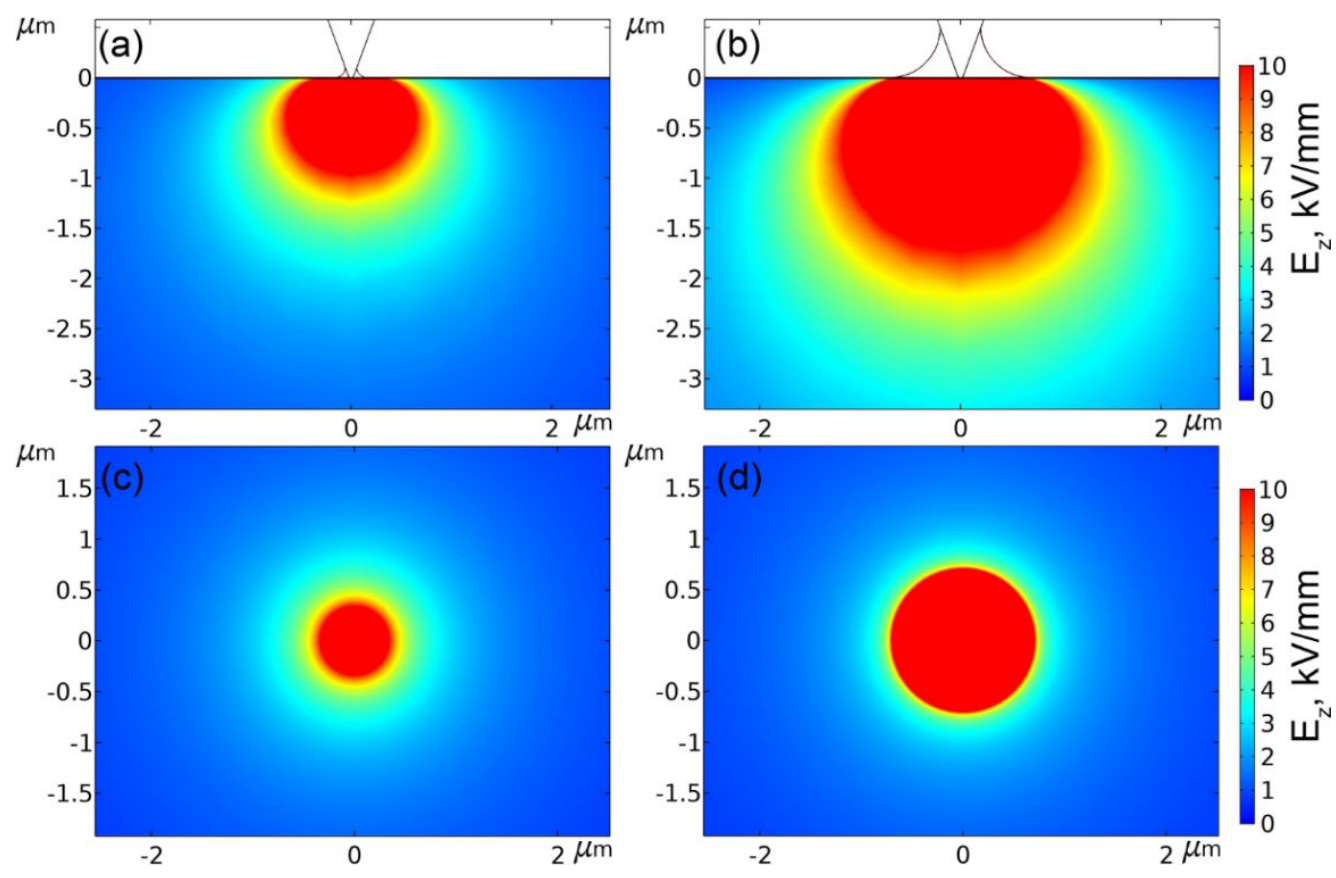

Figure S1. The calculated spatial distributions of the polar component of applied field $E_{z}$

for different radii of the water meniscus: $(a, c) 100 \mathrm{~nm}$, (b,d) $500 \mathrm{~nm}$. (a,b) ZY crosssections, (c,d) XY cross-sections at $10 \mathrm{~nm}$ below the polar surface.

S2. Calculation of the time dependence of the domain length (elongation) for local switching by a biased tip with a water meniscus

The domain growth by the fast wall motion (elongation) for switching by a biased tip with a water meniscus was simulated by COMSOL Multiphysics with PDE interface using calculated $E_{z}$ spatial distribution. The following dependence of the fast wall motion velocity $v$ on $E_{z}(X)$ excess over the threshold value $E_{\text {th.f }}$ was used: 


$$
v(X)=\mu_{f}\left[E_{z}(X)-E_{t h . f}\right]
$$

where $E_{\text {th.f }}=4 \mathrm{kV} / \mathrm{mm}$ (experimental value obtained for switching in uniform field (Ref. 18

in the manuscript)), $\mu_{f}$ - domain wall mobility, $X$-distance from the tip to the wall.

To calculate the time dependence of domain wall length we solved numerically the

following differential equation using Newton's method:

$$
\frac{d X}{d t}=v(X(t))
$$

where $\mathrm{X}$ is a domain wall position.

The boundary condition:

$$
X\left(t_{s t}\right)=L_{s t} / 2
$$

where $L_{s t}$ is the initial domain size, $t_{s t}$ is the start time of domain wall motion.

The obtained equation was used for approximation of the initial part of the experimental time dependences of the domain length $L(t)$ for humid conditions (Fig.3d) with meniscus size $\left(R_{w}\right)$ as a free parameter. The used values of $t_{\mathrm{st}}$ and $L_{\mathrm{st}}$ parameters were equal to the pulse duration and domain length for the lowest pulse duration, respectively. The used values of the parameters $E_{\text {th.f }}$ and $\mu_{f}$ correspond to the previously reported values for switching in uniform electric field (Ref. 18 in the manuscript). 
S3. The domain growth limited by the high-resistivity surface water layer

The existence of the high-resistivity surface water layer leads to a further domain growth

outside the area covered by the meniscus. The time dependence of the domain length

for local switching by the biased tip has been considered as a current limited switching

process. The surface water layer acts as a high-resistivity electrode. The switched bound

charge $Q_{b}$ is compensated by the external screening charge $Q_{s c r}$. For complete screening:

$$
Q_{b}-Q_{s c r}=A\left(P_{s}-\sigma_{s c r}\right)=0
$$

where $A$ is the switched area, $P_{s}$ is the spontaneous polarization, $\sigma_{s c r}$ is the screening charge density.

The current through the electrode $j_{s}(t)$ during switching by the domain wall motion

$$
j_{S}(t)=2 P_{s} \frac{d A}{d t}=2 P_{s} 2 w \frac{d X}{d t}
$$

where $w$ is the domain width.

At the same time, the screening current depends on the resistance of the electrode between the conductive tip and the moving domain wall

$$
j_{s c r}(t)=\frac{2\left(U_{t i p}-U_{t h . f}\right)}{R(t)}=\frac{2 w\left(U_{t i p}-U_{t h . f}\right)}{\rho X(t)}
$$


where $U_{t i p}$ is the applied voltage, $U_{t h . f i s}$ the threshold voltage for fast domain wall, $R(t)=\rho$

$\mathrm{X}(\mathrm{t}) / \mathrm{w}$ is the resistance of the surface layer, $\rho$ is the sheet resistance of the surface layer.

For $j_{s}(t)=j_{s c r}(t)$ we get the following relation:

$$
2 P_{S} 2 w \frac{d X}{d t}=\frac{2 w\left(U_{t i p}-U_{t h . f}\right)}{\rho X(t)}
$$

After simple transformations we obtain:

$$
\begin{gathered}
X(t) d X=\frac{U_{t i p}-U_{t h . f}}{2 P_{s} \rho} d t \\
\frac{X^{2}(t)}{2}=\frac{\left(U_{t i p}-U_{t h . f}\right) t}{2 P_{s} \rho} \\
X(t)=\left[\frac{\left(U_{t i p}-U_{t h . f}\right) t}{P_{s} \rho}\right]^{0.5}
\end{gathered}
$$

Finally, we obtain the following time dependence for the domain length:

$$
L(t)=L_{0}+2 X(t)=L_{0}+2\left[\frac{\left(U_{t i p}-U_{t h . f}\right) t}{P_{s} \rho}\right]^{0.5}
$$

\section{S4. Approximation details}

Here we provide the fitting parameters for all the curves presented in the manuscript text.

\section{S4.1 Voltage dependences of the domain length and width (Fig.2c-f)}

The measured data voltage dependences of the domain length and width were fitted with

the following formulas, which are typical for local switching in uniaxial ferroelectrics:

$$
L(U)=\frac{d L}{d U}\left(U-U_{t h . f}\right)
$$




$$
w(U)=\frac{d w}{d U}\left(U-U_{t h . s}\right)
$$

where $U_{\text {th.f }}$ and $U_{\text {th.s }}$ are the threshold voltages for the motion of fast and slow walls respectively, $d L / d U$ and $d w / d U$ are the coefficients characterizing the rate of domain growth with the voltage increase.

The fitting parameters are presented in Table S1. The obtained increase in the slopes $d L / d U$ and $d w / d U$ and the threshold voltages $U_{\text {th.f }}$ and $U_{\text {th.s }}$ for both dependences can be attributed to two mechanisms: (1) appearance of the water meniscus, which increases the field at the distance from the tip ranging from $200 \mathrm{~nm}$ to the meniscus radius and (2) formation of the water layer, which increases the wall motion velocity due to acceleration of the external screening at the distance from the tip above the meniscus radius.

Table S1 Fitting parameters for the voltage dependences of domain length and width for dry atmosphere and $\mathrm{RH}=30 \%$.

\begin{tabular}{|c|c|c|c|}
\hline & $\mathrm{RH}, \%$ & 0 & 30 \\
\hline \multirow{3}{*}{ Length } & $\mathrm{dL} / \mathrm{dU}, \mu \mathrm{m} / \mathrm{V}$ & 0.0043 & 0.0081 \\
\cline { 2 - 4 } & $\mathrm{U}_{\text {th.f, }} \mathrm{V}$ & 4.7 & 12.3 \\
\hline \multirow{2}{*}{ Width } & $\mathrm{dw} / \mathrm{dU}, \mu \mathrm{m} / \mathrm{V}$ & 0.0026 & 0.0063 \\
\cline { 2 - 4 } & $\mathrm{U}_{\text {th.s. }}, \mathrm{V}$ & 19 & 20 \\
\hline
\end{tabular}

S4.2 Pulse duration dependence of the domain length (Fig. 3d) 


\section{Dry atmosphere}

The experimental data fitting was based on the numerical calculation of spatial distribution

of the polar component of the electric field (see Section S1) taking into account the temporal decrease in the threshold field due to slow bulk screening processes (Eq. 4).

The fitting parameters are presented in the Table S2.

Table S2 The fitting parameters for pulse duration dependence of the domain length for dry atmosphere.

\begin{tabular}{|l|l|}
\hline$E_{t h}{ }^{0}, \mathrm{kV} / \mathrm{mm}$ & 4.2 \\
\hline$E_{t h}{ }^{m i n}, \mathrm{kV} / \mathrm{mm}$ & 2.4 \\
\hline$\tau, \mathrm{s}$ & 28 \\
\hline
\end{tabular}

\section{Humid conditions}

In this case, we used two models for fitting of the experimental data. The initial part of the

dependence was fitted by numerical solution of Eq. S2 based on the calculated spatial field distribution $E_{Z}(X)$ (section S1). The final part of the dependence was fitted by the formula for the domain growth limited by the surface water layer conductivity (Eq. S11):

$$
L(t)=L_{0}+A \times\left(t-t_{0}\right)^{0.5}
$$

where $A=2\left[\left(U_{t i p}-U_{t h . f}\right) /\left(\rho P_{S}\right)\right]^{0.5}, t_{0}$ and $L_{0}$ are the moment of time and domain length corresponding to the transition from the first model to the second one. 
The fitting parameters are presented in Table S3.

Table S3 The fitting parameters for pulse duration dependence of domain length for humid conditions

\begin{tabular}{|c|c|c|c|c|c|c|c|c|}
\hline Part & \multicolumn{4}{|c|}{ Initial part } & \multicolumn{4}{c|}{ Final part } \\
\hline Formula & \multicolumn{2}{|c|}{ Eq. S2 for calculated $E_{z}(X)$} & \multicolumn{3}{c|}{ Eq. S14 } \\
\hline $\mathrm{RH}, \%$ & & 15 & 50 & 70 & & 15 & 50 & 70 \\
\hline \multirow{3}{*}{$\begin{array}{c}\text { Parameter } \\
\mathrm{s}\end{array}$} & $R_{w}, \mathrm{~nm}$ & 340 & 600 & 980 & $L_{0}, \mu \mathrm{m}$ & 1.31 & 1.85 & 2.72 \\
\cline { 2 - 9 } & $\mu_{\mathrm{f}}, 10^{-14} \mathrm{~m}^{2} /(\mathrm{V} \cdot \mathrm{s})$ & 1.5 & 1.5 & 3.5 & $\begin{array}{c}A, \\
\mu \mathrm{m} / \mathrm{s}^{0.5}\end{array}$ & 0.044 & 0.07 & 0.12 \\
\cline { 2 - 9 } & $E_{\text {th.f. }} \mathrm{kV} / \mathrm{mm}$ & 4 & 4 & 4 & $t_{0}, \mathrm{~s}$ & 10 & 2 & 1 \\
\hline
\end{tabular}

The meniscus radius obtained from the fitting of the initial part increases with $\mathrm{RH}$ which

can be related to the obvious increase in the adsorbed water layer thickness.

Almost threefold increase in $A$ parameter with $\mathrm{RH}$ is obtained during fitting of the final part. As $U_{t i p}, U_{t h . f,}$ and $P_{S}$ are independent on $\mathrm{RH}$, this increase can be explained by the 9 times decrease in the sheet resistance of the water layer $\rho$ which, in turn, can be related to the corresponding increase in the water layer thickness.

As the transition to the water layer limited growth model occurs for domain lengths higher than the water meniscus radius, the increase in $\mathrm{RH}$ leads to the increase in $L_{O}$ parameter. 
The increase in domain wall velocity with $\mathrm{RH}$ at the initial part due to increase in meniscus radius leads to decrease in $t_{O}$ parameter.

\section{S4.3 Pulse duration dependence of domain width (Fig. 3e)}

The domain widening caused by the motion of the slow domain walls is comparatively slow, so its velocity is limited by the wall mobility, but not by the water layer conductivity.

The following time dependence of the domain width is obtained for the linear voltage dependence of the domain wall velocity:

$$
w(t)=w_{0}+v_{s} t
$$

where $w_{o}$ is the width of the initial domain, $v_{s}(U)=2 k_{w}\left(U_{t i p}-U_{t h . s}\right), k_{w}$ is the coefficient, Uth.s is the threshold voltage for the slow domain wall.

The fitting results are presented in the Table S4.

Table S4 The fitting parameters for pulse duration dependence of the domain width.

\begin{tabular}{|c|c|c|c|c|}
\hline $\mathrm{RH}, \%$ & 0 & 15 & 50 & 70 \\
\hline $\mathrm{w}_{0}, \mu \mathrm{m}$ & 0.47 & 0.71 & 0.80 & 0.97 \\
\hline $\mathrm{v}_{\mathrm{s}}, \mu \mathrm{m} / \mathrm{s}$ & 0.009 & 0.011 & 0.008 & 0.012 \\
\hline
\end{tabular}


The increase in $w_{0}$ parameter can be related to increase in the meniscus radius with $\mathrm{RH}$ which determines the initial domain size. As the domain wall motion is governed by the mobility of the domain wall, but not by the water layer conductivity, the domain wall velocity is independent (within the measurement accuracy of about $0.002 \mu \mathrm{m} / \mathrm{s}$ ) on RH. 